

\section{LA VIOLENCIA EN REDES SOCIALES: UN ANÁLISIS A PARTIR DE FACTORES CONTEXTUALES E INDIVIDUALES}

VIOLENCE IN SOCIAL NETWORKS: AN ANALYSIS

BASED ON CONTEXTUAL AND INDIVIDUAL FACTORS

\section{ANA KAREN GUTIÉRREZ MARTIÍNEZ}

\section{RESUMEN}

El ciberacoso es un problema actual, presente en la población adolescente, que tiene consecuencias negativas en la vida de los jóvenes. En este estudio se pretende estimar y contrastar un modelo de efectos directos e indirectos de la Cibervictimización en adolescentes de Nivel Medio Superior que integre variables Personales (Autoestima, Malestar Psicológico, Reputación Social) y Contextuales (Comunicación Familiar, Funcionamiento Familiar, Clima Social en el Aula y Adicción a Redes Sociales). Se aplicaron las escalas de Victimización Virtual (Buelga, Cava y Musitu, 2012), AF-5 (García y Musitu, 1999), Malestar Psicológico (Kessler \& Mroczek, 1999), Reputación Social (Caroll, Houghton, Hattie, \& Durkin, 1999), Comunicación Familiar (Barnes \& Olson, 1982, 1985), Funcionamiento Familiar (Smilkstein,Ashworth, \& Montano, 1982), Clima Social en el Aula (Moos, Moos \& Trickett, 1984) y Adicción a Redes Sociales (Peris, Maganto, \& Garaigordobil, 2018) a una población de 1306 adolescentes de un plantel educativo ubicado en el noreste del estado de Nuevo León. Los resultados preliminares, correspondientes al primer objetivo específico del estudio, y obtenidos a través de un análisis de correlación de Pearson, muestran que las variables personales y contextuales están relacionadas de forma significativa con la victimización virtual de los jóvenes. Dicho hallazgo resalta la importancia de tomar en cuenta a la familia y la escuela en las líneas de acción preventivas y de intervención de la problemática.

PALABRAS CLAVE: CIBERVICTIMIZACIÓN, ACOSO, ADOLESCENTES, PERSONALES, CONTEXTUALES, VIRTUAL.

\section{ABSTRACT}

Cyberbullying is a current problem, present in the adolescent population, which has negative consequences on the lives of young people. This study aims to estimate and contrast a model of direct and indirect effects of cybervictimization in adolescents of upper middle level that integrates personal (self-esteem, psychological discomfort, social reputation) and contextual variables (family communication, family functioning, social climate in the classroom and social media addiction). The scales of virtual victimization were applied (Buelga, Cava and Musitu, 2012), AF-5 (García and Musitu, 1999), psychological distress (Kessler \& Mroczek, 1999), social reputation (Caroll, Houghton, Hattie, \& Durkin, 1999), family communication (Barnes \& Olson, 1982, 1985), family functioning (Smilkstein, Ashworth, \& Montano, 1982), social climate in the classroom (Moos, Moos \& Trickett, 1984) and addiction to social networks (Peris, Maganto, \& Garaigordobil, 2018) to a population of 1306 adolescents from an educational campus located in the northeast of the state of Nuevo León. Preliminary results, corresponding to the first specific objective of the study, and obtained through a Pearson moment product correlation analysis, show that personal and contextual variables are significantly related to the virtual victimization of young people. This finding highlights the importance of taking family and school into account in the lines of preventive action and intervention of the problem.

KEYWORDS: CYBERVICTIMIZATION, HARASSMENT, ADOLESCENTS, PERSONAL, CONTEXTUAL, VIRTUAL. 
I ciberacoso es una nueva forma de intimidación entre adolescentes caracterizado por ataques reiterados, difamaciones $u$ otras formas de hostigamiento a través de dispositivos electrónicos como el celular (Buelga \& Pons, 2012).

La comunidad científica se ha interesado en estudiar las diferencias de prevalencia de cibervictimización en hombres y mujeres. Hay evidencia empírica de que las mujeres son más victimizadas que los varones, pero también existen estudios que demuestran lo contrario o que no encuentran diferencias significativas entre ambos (Buelga, Cava, \& Musitu, 2010; Tokunaga, 2010).

Asimismo, la cibervictimización ha sido asociada a episodios prolongados de malestar psicológico que incluye la presencia de estrés, angustia, depresión y baja autoestima en los adolescentes (Bayraktar, Machackova, Dedkova, Cerna, \& Ševčíková, 2015; Garaigordobil, 2015).

En lo tocante a la familia y la escuela, Órtega-Barón (2016) encontraron que las cibervíctimas tienen más conflictos familiares, una baja autoestima académica y menor implicación escolar. Asimismo, Buelga, Martínez Ferrer, \& Cava (2017) reportaron que los adolescentes víctimas de cyberbullying perciben una comunicación menos abierta con su madre y una comunicación evitativa con su padre.

Existen estudios que asocian el uso frecuente de internet, entendido como un riesgo de adicción a redes sociales, con la prevalencia de cibervictimización (Álvarez García, Nuñez Pérez, Dobarro González, \& Rodrí-
Hay evidencia empírica de que las mujeres son más victimizadas que los varones, pero también existen estudios que demuestran lo contrario o que no encuentran diferencias significativas entre ambos

$\infty<\infty<\infty<\infty<\infty<\infty<\infty<\infty<\infty<\infty<\infty<\infty<\infty<\infty<\infty<\infty<\infty<\infty<\infty<\infty<\infty<\infty<\infty<\infty<\infty<\infty<\infty<\infty<\infty$

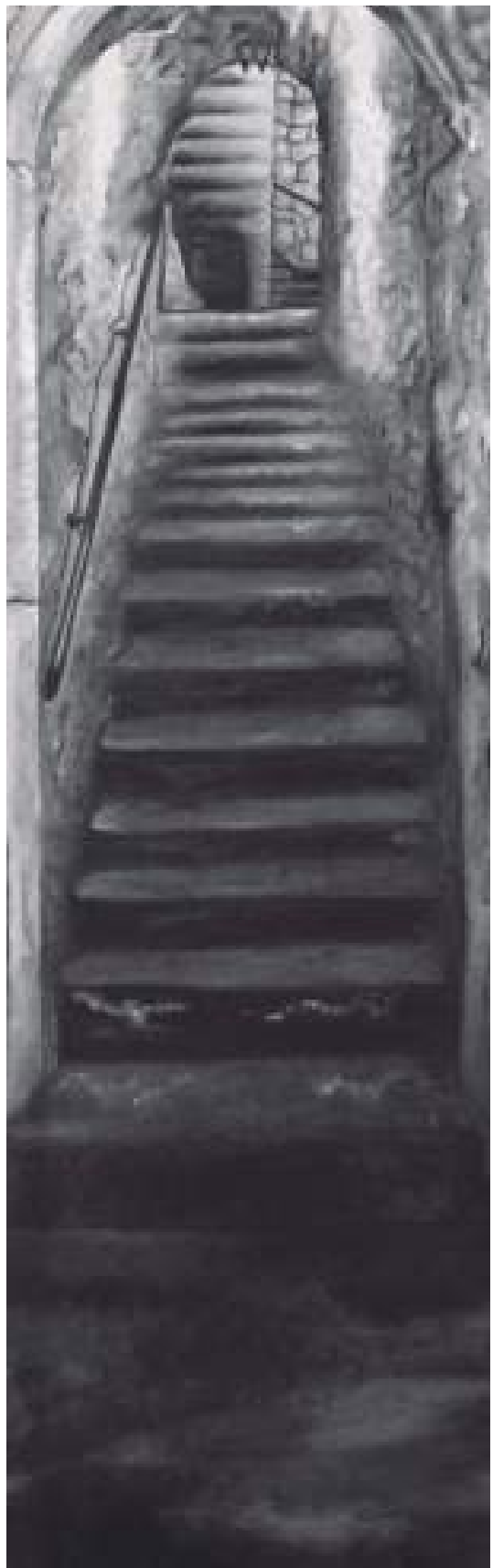

guez Pérez, 2015; Didden et al., 2009; Twyman, Saylor, Taylor, \& Comeaux., 2010; Ybarra \& Mitchell, 2004).

\section{PLANTEAMIENTO DEL PROBLEMA}

El ciberacoso es un problema actual en la población adolescente que tiene consecuencias negativas en la vida de los jóvenes. En diversos estudios internacionales se reportó una prevalencia significativa de ciberacoso: 35.7\% en Turquía (Beyazit, Şimsek, \& Ayhan, 2017), 27.4\% en España (Ortega-Barón, et al. 2016) y 29.4\% en Estados Unidos (Patchin, \& Hinduja, 2010). En países latinoamericanos como Colombia, Chile y Perú existen riesgos como el de ser contactados sin quererlo o recibir mensajes con contenido sexual no deseado (Microsoft, 2018).

En México, la prevalencia es de $25.4 \%$ en varones y $28.1 \%$ en mujeres, de acuerdo a un estudio realizado por el Instituto Nacional de Estadística y Geografía (INEGI, 2015).

Ante esta situación, y por su potencial dañino, la comunidad científica se ha interesado en conocer los factores relacionados a los episodios de cyberbullying. No obstante, la mayor 
FIGURA 1. Variables de Estudio (Personales y Contextuales)

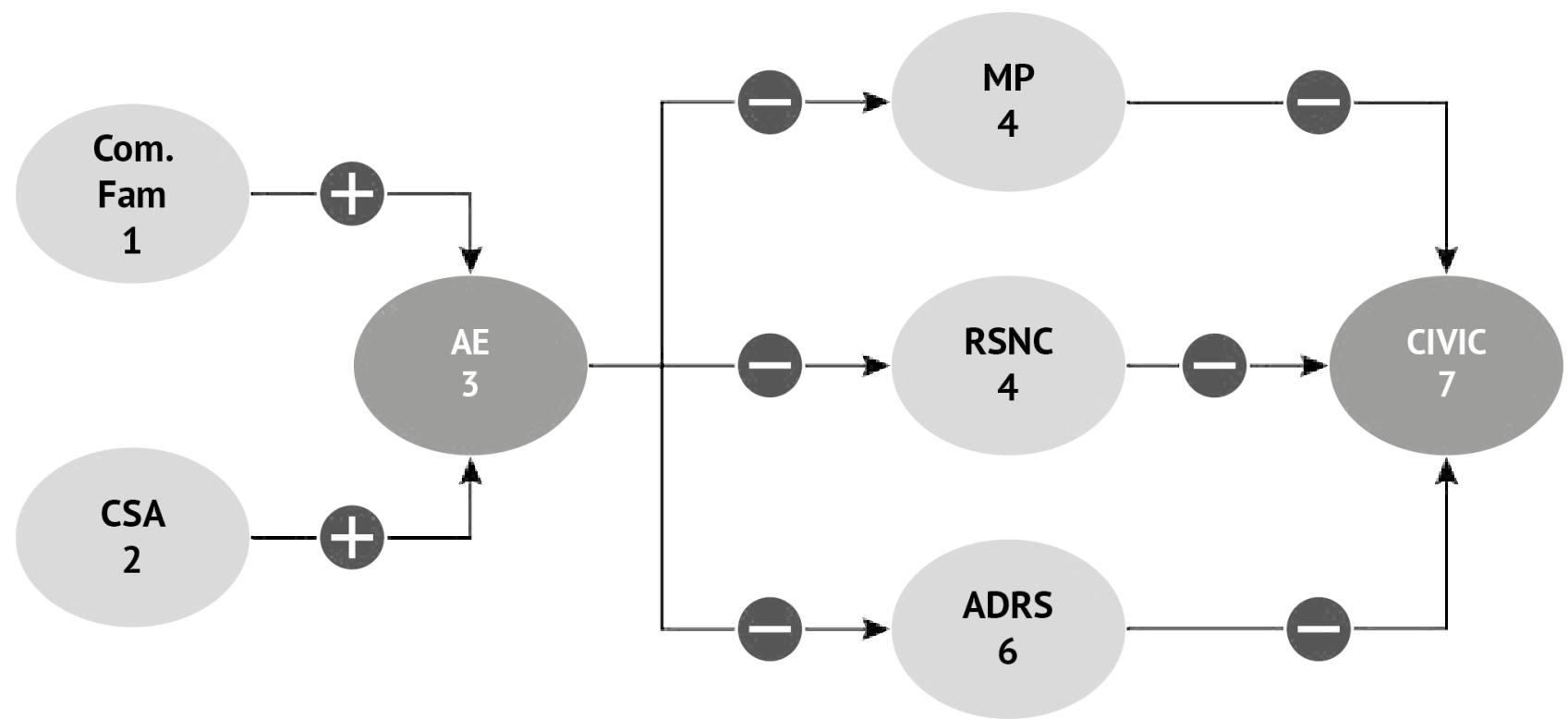

parte de los trabajos se han realizado en países europeos, Estados Unidos y Canadá, siendo fundamental realizar un estudio de naturaleza explicativa en México y, concretamente, en el Estado de Nuevo León.

A partir de lo anterior, nos preguntamos: ¿Cuál es la influencia de las variables personales y contextuales en la cibervictimización en adolescentes de Nivel Medio Superior? Con ello, buscamos aportar elementos teórico-metodológicos para la explicación del fenómeno de ciberacoso en adolescentes neoloneses y así ayudar

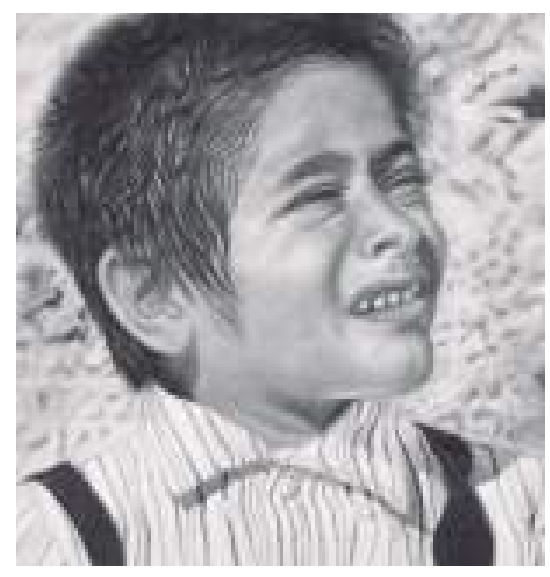

a delinear acciones de prevención e intervención en la problemática.

\section{OBJETIVO GENERAL \\ DE LA INVESTIGACIÓN}

Estimar y contrastar un modelo predictivo de efectos directos e indirectos de la cibervictimización en adolescentes que integre variables personales y contextuales.

Los objetivos específicos de la investigación son los siguientes:

- Analizar la relación existente entre la cibervictimización y las variables de estudio.
- Contrastar un modelo predictivo de la cibervictimización que integre variables personales $y$ contextuales.

- Analizar en función del sexo las relaciones entre la cibervictimización y las variables consideradas en el estudio.

- Diseñar un programa de prevención e intervención de la cibervictimización a partir del modelo contrastado.

Las hipótesis son las siguientes:

1. Existe una relación significativa entre la cibervictimización y las 


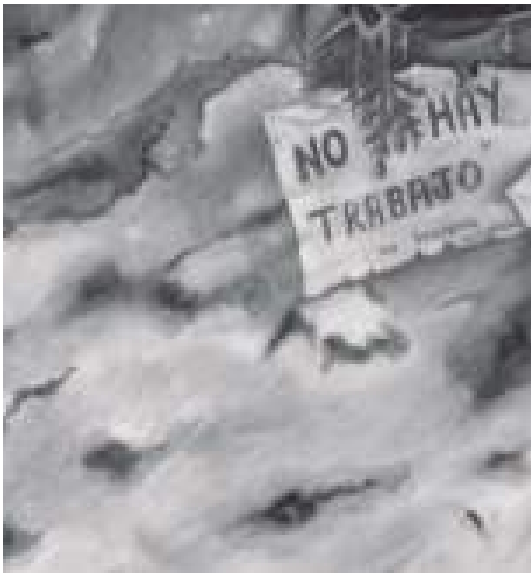

variables de estudio (personales y contextuales).

2. Si existe una comunicación familiar abierta y un clima social positivo en el aula, el adolescente presentará un buen nivel de autoestima, lo que a su vez incide en la ausencia de malestar psicológico, reputación social no conformista y adicción a redes sociales, variables que a su vez disminuye la probabilidad de que haya cibervictimización. Si existe una comunicación familiar ofensiva y evitativa y un clima social negativo en el aula, el adolescente presentará un bajo nivel de autoestima, lo que

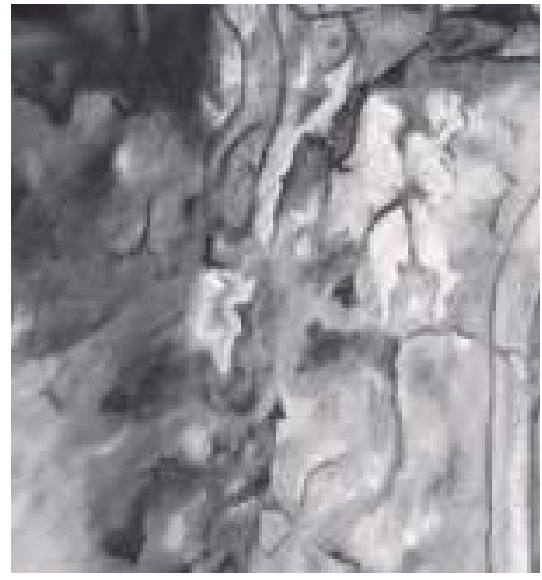

a su vez incide en la aparición de malestar psicológico, una reputación social no conformista, y adicción a redes sociales, variables que a su vez aumentan la probabilidad de que haya cibervictimización.

3. La relación entre la cibervictimización y las variables del estudio en función del sexo es igual, con excepción de las variables de malestar psicológico y reputación social. Las mujeres presentan mayor malestar psicológico y los varones mayor reputación social no conformista, las cuales tienen un efecto directo en la cibervictimización.

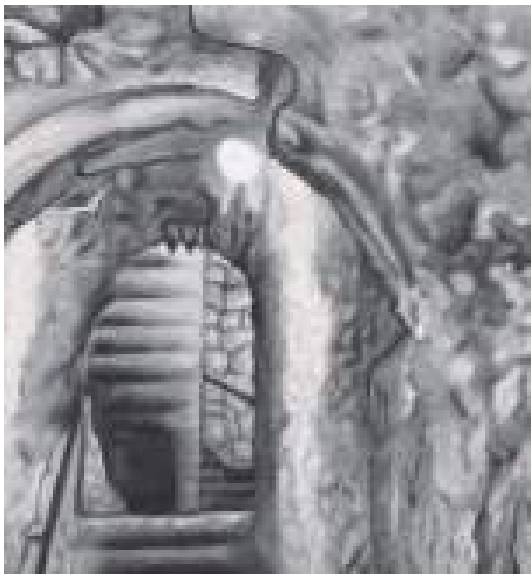

\section{JUSTIFICACIÓN}

\section{DE LA INVESTIGACIÓN}

Dado que el cyberbullying es un problema que aqueja a los niños y jóvenes desde temprana edad (UNESCO, 2017), resulta relevante realizar este trabajo pues contribuye a comprender la etiología del fenómeno de ciberacoso y aporta elementos para construir y orientar proyectos de intervención, prevención y psicoeducación enmarcados en el tema de la violencia virtual y el acoso escolar.

Tal y como se mencionó en el planteamiento del problema, no existe en territorio neolonés un modelo explicativo del ciberacoso en adolescen-

TABLA 1.Análisis Descriptivo Muestral

\begin{tabular}{|c|c|c|c|c|}
\hline \multirow{2}{*}{ Sexo } & Hombre & N & \% & \% VÁLIDO \\
\hline \multirow{2}{*}{ Semestre } & Mujer & 643 & 50 & 50.3 \\
\cline { 2 - 5 } & Segundo & 666 & 49.4 & 49.7 \\
\cline { 2 - 5 } & Cuarto & 636 & 41.0 & 51.2 \\
\hline \multirow{2}{*}{ Clasificación } & Tempranos (12-14) & 0 & 0 & 48.8 \\
\hline Adolescencia & Medios (15-17) & 1294 & 99.1 & 0 \\
\cline { 2 - 5 } & Tardíos (18-21) & 9 & 0.7 & 99.1 \\
\hline
\end{tabular}


TABLA 2. Instrumentos de Recolección de Datos

\begin{tabular}{|c|c|c|c|}
\hline NOMBRE & MIDE & AUTORES & ADAPTACIÓN AL ESPAÑOL \\
\hline $\begin{array}{c}\text { Escala de Victimización } \\
\text { Virtual }\end{array}$ & $\begin{array}{c}\text { Victimización } \\
\text { en Redes Sociales }\end{array}$ & $\begin{array}{l}\text { Buelga, Cava y Musitu } \\
\text { (2012) }\end{array}$ & No Aplica \\
\hline $\begin{array}{l}\text { Escala de Malestar } \\
\text { Psicológico }\end{array}$ & Malestar Psicológico & $\begin{array}{c}\text { Kessler y Mroczek } \\
(1994)\end{array}$ & Lisis México \\
\hline $\begin{array}{l}\text { Escala de Reputación } \\
\text { Social }\end{array}$ & $\begin{array}{l}\text { Reputación Social } \\
\text { No Conformista }\end{array}$ & $\begin{array}{l}\text { Carroll, Houghton, } \\
\text { Hattie y Durkin (1999) }\end{array}$ & Lisis México \\
\hline $\begin{array}{l}\text { Escala de Riesgo de } \\
\text { Adicción a Redes Sociales }\end{array}$ & $\begin{array}{l}\text { Adicción a Redes } \\
\text { Sociales }\end{array}$ & $\begin{array}{c}\text { Peris, Maganto } \\
\text { y Garaigordobil (2018) }\end{array}$ & No Aplica \\
\hline AF-5 & $\begin{array}{l}\text { Autoestima } \\
\text { de Protección }\end{array}$ & $\begin{array}{c}\text { García y Musitu } \\
\text { (1999) }\end{array}$ & No Aplica \\
\hline $\begin{array}{c}\text { Escala de Comunicación } \\
\text { Familiar }\end{array}$ & $\begin{array}{l}\text { Comunicación con la } \\
\text { Madre y el Padre }\end{array}$ & $\begin{array}{l}\text { Barnes y Olson } \\
(1982,1985)\end{array}$ & Lisis México \\
\hline $\begin{array}{l}\text { Escala de Clima } \\
\text { Social en el Aula }\end{array}$ & $\begin{array}{l}\text { Implicación, Apatía y } \\
\text { Rechazo Escolar }\end{array}$ & $\begin{array}{c}\text { Moos, Moos y Trickett } \\
\text { (1984) }\end{array}$ & Lisis México \\
\hline $\begin{array}{c}\text { Escala de Funcionamiento } \\
\text { Familiar }\end{array}$ & $\begin{array}{c}\text { Funcionamiento } \\
\text { Familiar }\end{array}$ & $\begin{array}{l}\text { Smilkstein, Ashworth } \\
\text { y Montano (1982) }\end{array}$ & $\begin{array}{l}\text { Bellon, Luna y Lardelli } \\
\text { (1996) }\end{array}$ \\
\hline
\end{tabular}

tes de Nivel Medio Superior, con lo cual se espera que esta investigación contribuya a llenar este vacío de conocimiento al poder conocer mejor la relación existente entre el cyberbullying y las variables personales y contextuales seleccionadas.

Lo anterior, no obstante, deberá considerarse dentro de las limitaciones del estudio ya que al no poder seleccionar aleatoriamente a los participantes, no se podrán generalizar los resultados, siendo pertinente realizar nuevas investigaciones en otros contextos también de México.

La presente investigación se desarrollará desde el Modelo Ecológico del Desarrollo de Urie Bronfenbrenner (1979), a través del cual se explica que el desarrollo de un individuo depende de una compleja interrelación

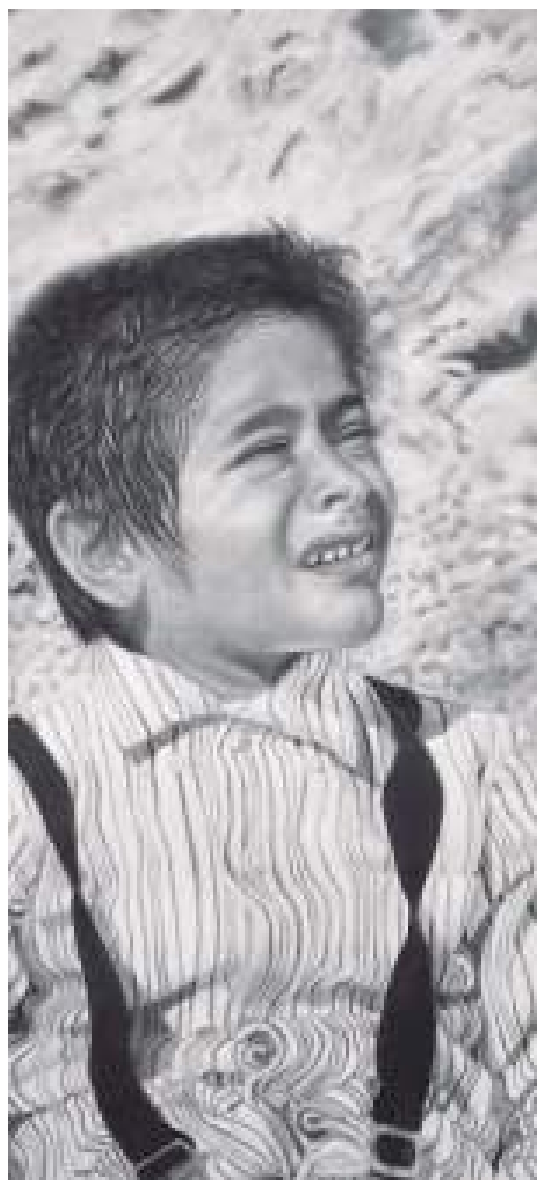

de factores (Meece, 2001). En el centro se encuentra el individuo, con sus características innatas temperamentales, físicas y mentales. Alrededor suyo interactúan 4 niveles, que constituyen una influencia significativa en su desarrollo.

El 1er nivel es el entorno más allegado conformado por la familia, la escuela y los compañeros, y constituye lo que Bronfenbrenner llamó microsistema, porque en él es posible una interacción cercana, cotidiana y diaria. El 2do nivel, llamado mesosistema, refleja las interrelaciones entre los individuos que conforman el microsistema, por ejemplo, relaciones de filiación, de trabajo o de amistad.

El 3er nivel, llamado exosistema, incluye elementos institucionales y socioeconómicos como por ejemplo 
el nivel académico de la escuela, los servicios de salud, el lugar de trabajo de los padres, entre otros, que en determinada manera influyen en las personas y en sus interrelaciones.

El 4to nivel, llamado macrosistema, constituye la cultura y/o subcultura en la que el individuo vive y se desarrolla, y abarca mayores elementos como la cosmovisión del mundo, el sistema de valores, las leyes y reglamentos, la religión, entre otros (Bronfenbrenner, 1979; Feldman, 2007; Meeci, 2001). De acuerdo a Bronfenbrenner, no se puede entender el desarrollo si no se consideran todos los aspectos del modelo de manera interrelacionada (Feldman, 2007).

Las fortalezas de esta perspectiva teórica radican en que ésta permite analizar la conducta como producto de una compleja interrelación de factores, y considera que el individuo es, a la vez, un agente activo y pasivo en los diversos contextos en los que actúa (Monreal-Gimeno, Poveda-
Las fortalezas de esta perspectiva teórica radican en que ésta permite analizar la conducta como producto de una compleja interrelación de factores, y considera que el individuo es, a la vez, un agente activo y pasivo en los diversos contextos en los que actúa

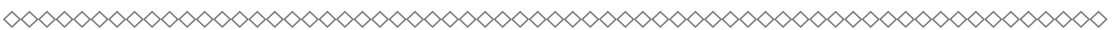

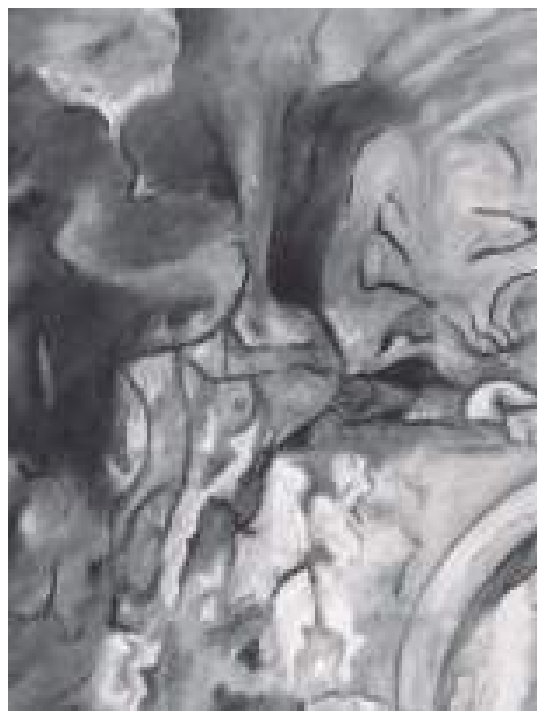

no-Díaz, \& Martínez-Ferrer, 2014). Al tomarla en cuenta en este trabajo, la teoría ecológica puede brindar una mayor comprensión del fenómeno de cibervictimización así como claves para la prevención e intervención de esta problemática social.

\section{PROCEDIMIENTO}

Al comienzo de la investigación se procedió a gestionar el permiso en el plantel educativo para la aplicación del instrumento. Se formó igualmente un equipo de trabajo conformado

TABLA 3. Análisis de Correlación Producto Momento de Pearson

\begin{tabular}{|c|c|c|c|c|c|c|c|c|}
\hline & VICI & MP & FF & COMOFMP & RENOCONR & AEPROT & CSA & ADRED \\
\hline VICI & 1 & & & & & & \\
\hline MP & $0.398^{* *}$ & 1 & & & & & \\
\hline FF & $-0.229^{* *}$ & $-0.378^{* *}$ & 1 & & & & \\
\hline COMOFMP & $0.247^{* *}$ & $0.352^{* *}$ & $-0.414^{* *}$ & 1 & & & \\
\hline RENOCONR & $0.347^{* *}$ & $0.196^{* *}$ & $-0.181^{* *}$ & $0.209^{* *}$ & 1 & & & \\
\hline AEPROT & $-0.238^{* *}$ & $-0.460^{* *}$ & $0.566^{* *}$ & $-0.426^{* *}$ & $-0.297^{* *}$ & 1 & & \\
\hline CSA & $-0.250^{* *}$ & $-0.334^{* *}$ & $0.292^{* *}$ & $-0.257^{* *}$ & $-0.214^{* *}$ & $0.381^{* *}$ & 1 & \\
\hline ADRED & $0.263^{* *}$ & $0.282^{* *}$ & $-0.104^{* *}$ & $0.155^{* *}$ & $0.239^{* *}$ & $-0.099^{* *}$ & $-0.159^{* *}$ & 1 \\
\hline
\end{tabular}


por 25 estudiantes de pregrado, quienes mostraron gran entusiasmo y colaboración. Una vez capacitados, se formaron subequipos de 2 personas, los cuales ingresaron en las aulas del plantel para la aplicación. Se tomaron en cuenta medidas de calidad en la recabación de datos (ausencia de profesor, cercanía y compañerismo, consentimiento informado, confidencialidad, caja buzón, descanso). Una vez recabados los datos, se capturaron en el software SPSS y se realizó un análisis de correlación producto momento de Pearson.

\section{RESULTADOS}

En la Tabla 3 se muestran resultados correspondientes al primer objetivo específico de la investigación.

\section{CONCLUSIÓN}

Hasta el momento, se ha constatado que las variables personales de malestar psicológico y autoestima tienen relación significativa con la cibervictimización, tal y como lo encontraron Bayraktar, Machackova, Dedkova, Cerna, \& Ševčíková (2015) y Garaigordobil (2015). Igualmente las variables de funcionamiento y comunicación familiar mantienen una relación significativa con los episodios de victimización virtual, lo que coincide con los hallazgos de Buelga, et al. (2017), Órtega-Barón, et al. (2016) y Larrañaga, Yubero, Ovejero y Navarro (2016).

Es fundamental además destacar un hallazgo inesperado: la relación significativa entre la reputación social no conformista y la cibervictimización, que en alguna manera contrasta con el supuesto conceptual de que solo los adolescentes que juegan el papel de acosadores buscan tener

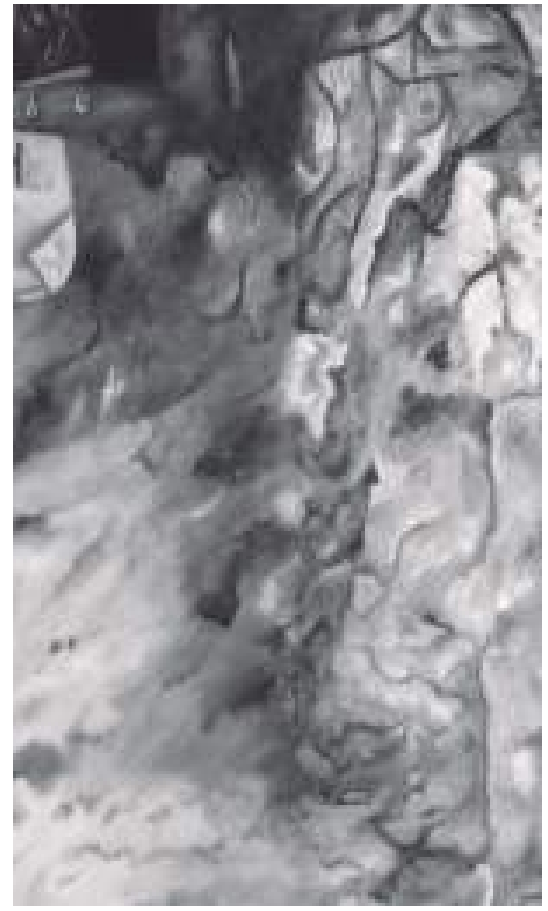

un reconocimiento social entre sus pares (Caroll, Houghton, Hattie, \& Durkin, 1999). Este descubrimiento podría ser explicado por el hecho de que los jóvenes cibervictimizados juegan también el papel de acosadores, siendo su rol ambivalente, volviéndose necesaria una mayor atención científica a dicha realidad (Pellegrini, Bartini, \& Brooks, 1999; Salmivalli y Nieminen, 2002; Cerezo, 2008).

\section{PROPUESTAS}

Los resultados presentados hasta el momento enfatizan el papel de los

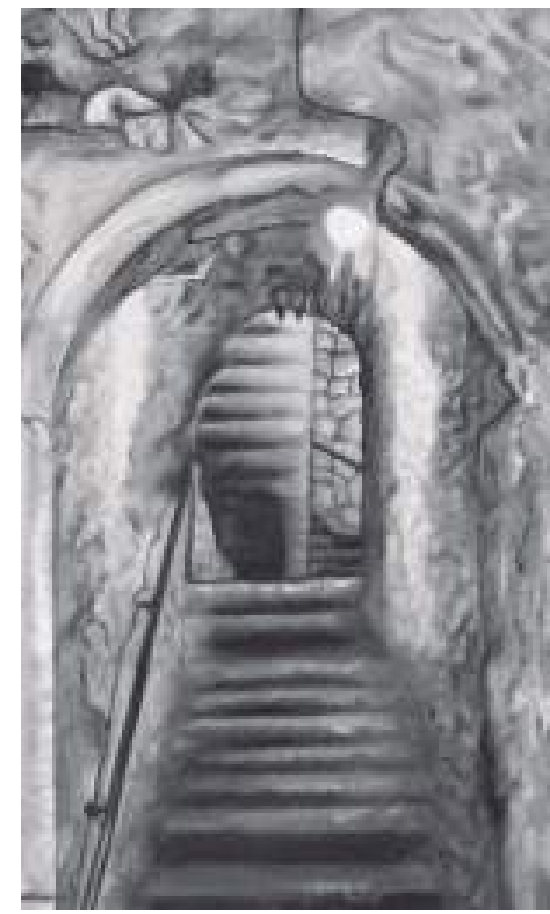

contextos escolar y familiar en el desarrollo de los adolescentes, dando pautas para tomar en cuenta estos escenarios a la hora de establecer acciones preventivas y de intervención en las situaciones problemáticas de los estudiantes.

De acuerdo a lo anterior, y una vez que se termine el estudio, podrá diseñarse un programa de prevención e intervención que integre variables contextuales y personales, y que enriquezca las acciones tomadas hasta el momento en materia de violencia en el ciberespacio.
Hasta el momento, se ha constatado que las variables personales de malestar psicológico y autoestima tienen relación significativa con la cibervictimización 


\section{REFERENCIAS}

Bayraktar, F., Machackova, H., Dedkova, L., Cerna, A., Ševčíková, A., (2015). Cyberbullying: The Discriminant Factors Among Cyberbullies, Cybervictims, and Cyberbully-Victims in a Czech AdolescentSample.Journal of interpersonalviolence 30(18).doi:10.1177/0886260514555006. Beyazit, U., Şimsek, Ş., Ayhan, A. (2017). An examination of the predictive factors of cyberbullying in adolescents. Social Behavior and personality: an international journal, 45(9), 1511-1522. doi: 10.2224/sbp6267.

Bronfenbrenner, U. (1987). La ecología del desarrollo humano. Barcelona, España: Paidós.

Buelga, S., Cava, M. J., y Musitu, G. (2010). Cyberbullying: victimización entre adolescentes a través del teléfono móvil y de Internet. Psicothema 22(4),784-789.

Buelga, S., Martínez-Ferrer, B., y Cava, M-J. (2017). Differences in Family Climate and Family Communication among Cyberbullies, Cybervictims and Cyber bully-victims in Adolescents. Computers in Human Behavior, doi: 10.1016/j.chb.2017.07.017.

Carroll, A., Houghton, S. Hattie, J. y Durkin, K. (1999). Adolescent reputation enhancement: differentiating delinquent, nondelinquent, and at-risk youths. Journal of Child Psychology and Psychiatry, 40, 593-606.

Cerezo, F. (2008). Agresores y víctimas del bullying. Desigualdades de género en la violencia entre escolares. Informacion Psicologica, 94, 49-59.

Feldman, R. (2007). Desarrollo Psicológico a través de la vida (4ta ed.). México: Pearson.

Garaigordobil, M. (2015). Psychometric properties of the cyberbullying test, a screening instrument to measure cybervictimization, cyberagression and cyberobservation. Journal of interpersonal violence 32(23). doi: 10.1177/0886260515600165.

Instituto Nacional de Estadística y Geografía (INEGI) (2016). Módulo sobre ciberacoso. MOCIBA 2015. Principales resultados.

Larrañaga, E., Yubero, S., Ovejero, A., Navarro, R. (2016). Loneliness, parent-child communication and cyberbullying victimization among Spanish youths. Computers in Human Behavior, 65, 1-8.

Meece, Y.L. (2001). Desarrollo del niño y del adolescente: Compendio para educadores (José Pecina, trad.). México: Mc.Graw-Hill Interamericana (Obra original publicada en 1997).

Microsoft (2018). Civility, Safety and Interaction online. Recuperado el 13 de septiembre de 2018 de https://query.prod.cms.rt.microsoft.com/cms/api/am/binary/RE1LWr6.

Monreal-Gimeno, M.C., Povedano-Díaz,A.,y Martínez-Ferrer, B.(2014). Modelo ecológico de los factores asociados a la violencia de género en parejas adolescentes. Journal for Educators, Teachers and Trainers, 5(3), 105-114.

Musitu, G., Buelga, S., Lila, M. \& Cava, M. (2001). Familia y adolescencia: Análisis de un modelo de intervención psicosocial. Madrid: Síntesis.

Órtega-Barón, J., Buelga, S., \& Cava, M-J. (2016). Influencia del clima escolar y familiar en adolescentes, víctimas de ciberacoso. Comunicar, XXIV(46), 57-65. doi: 10.3916/C46-2016-06. Patchin, J. W., \& Hinduja, S. (2010). Cyberbullying and Self-Esteem. Journal of School Health, 80(12), 614-621. doi: epdf/10.1111/j.1746-1561.2010.00548.x.

Pellegrini, A., Bartini, M. y Brooks, F. (1999). School bullies, victims, and aggressive victims: factors relating togroup affiliation and victimization in early adolescence. Journal of Educational Psychology, 91, 216-224.

Salmivalli, C.y Nieminen, E. (2002). Proactive and Reactive Aggression Among School Bullies, Victims, and Bully-Victims. Aggressive Behavior 28, 30-44. doi:10.1002/ab.90004.

Sánchez Sosa, J. C., Villarreal González, M. E., \& Musitu, G. (2019). La cibervictimización en adolescentes escolarizados: un análisis a partir de factores individuales y contextuales. Artículo en preparación.

Schultze-Krumbholz, A., Hess, M., Pfetsch, J., \& Scheithauer, H. (2018). Who is involved in cyberbullying? Latent class analysis of cyberbullying roles and their associations with aggression, self-esteem, and empathy. Cyberpsychology: Journal of Psychosocial Research on Cyberspace, 12(4), doi: 10.5817/CP2018-4-2.

Tokunaga, R. S. (2010). Following you home from school: a critical review and synthesis of research on cyberbullying victimization. Computers in Human Behavior, 26, 277-287. doi: 10.1016/j.chb.2009.11.014.

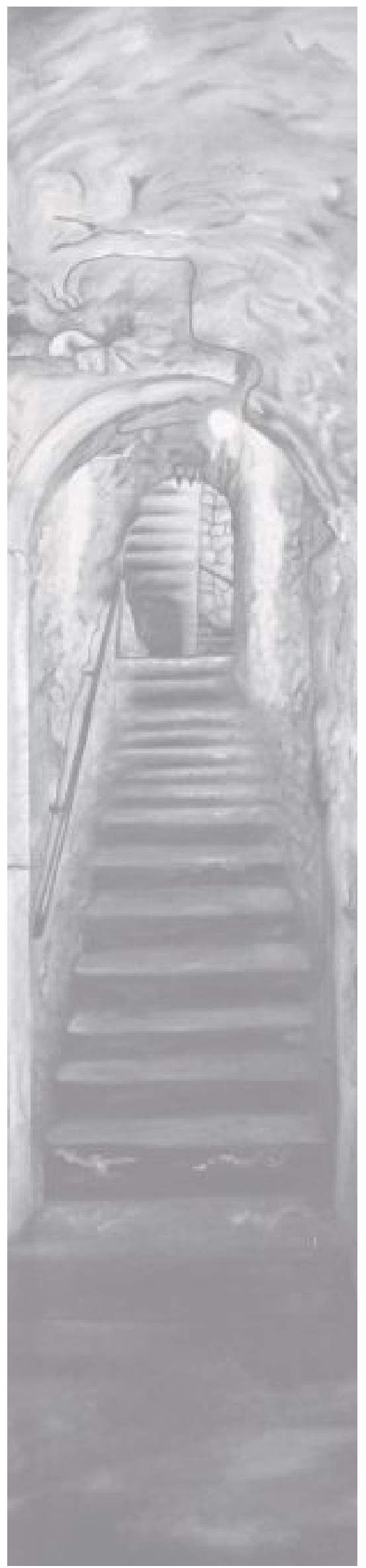




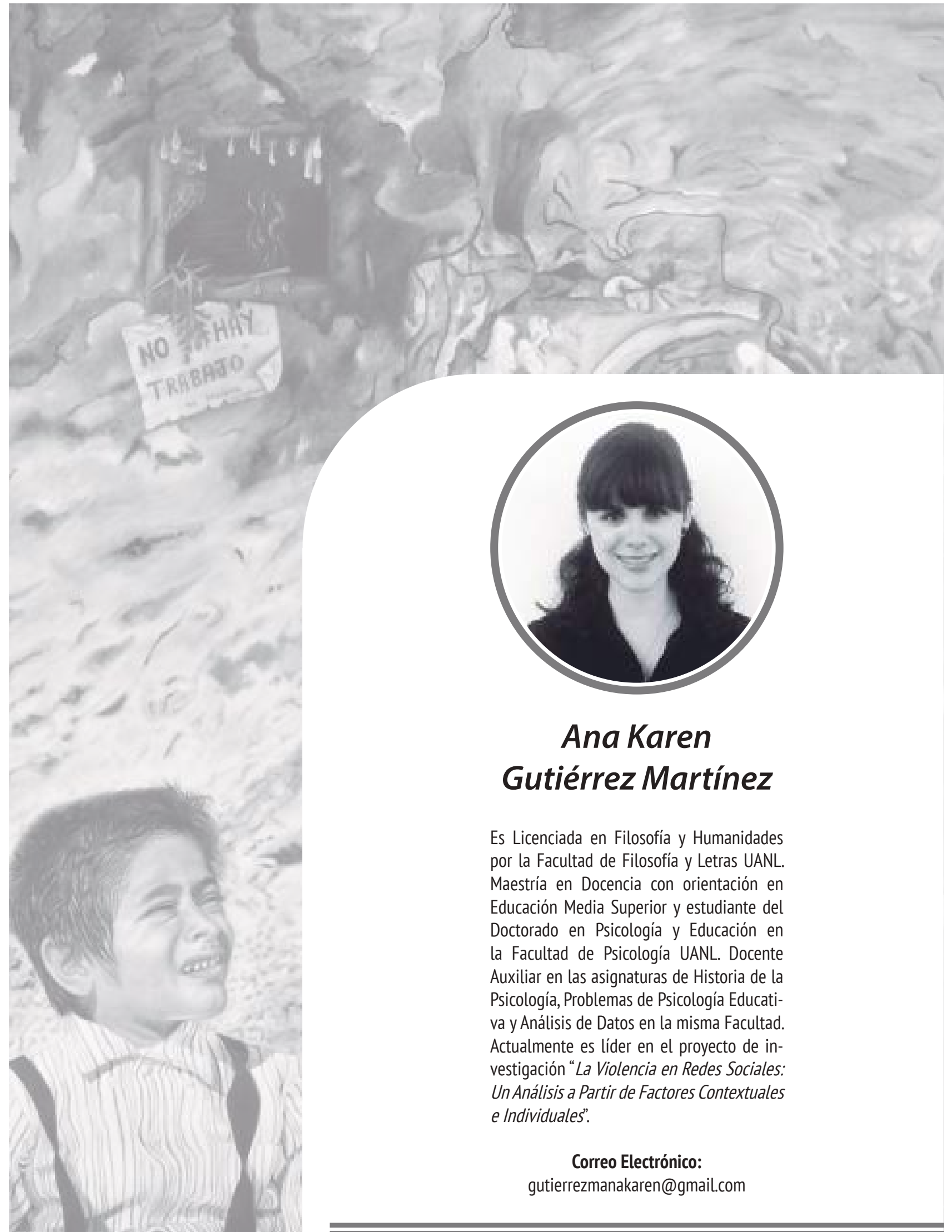

Recibido: 15/09/2019

Aceptado: 15/10/2019 